\title{
Hybrid Neuro-Fuzzy System for Optimal Generation scheduling in Electrical Power systems
}

\author{
Ruchi Solanki ${ }^{1}$, Krishna Teerth Chaturvedi ${ }^{2}$, Narayan Prasad. Patidar ${ }^{3}$ \\ ${ }^{I}$ Deptt. Of Electrical Engg., National Institute of Technology, Bhopal, India \\ ${ }^{2}$ Deptt. Of Electrical \& Electronics Engg. UIT-RGPV, Bhopal, India \\ ${ }^{3}$ Deptt. Of Electrical Engg. National Institute of Technology, Bhopal, India
}

\begin{abstract}
The real time controls at the central energy management center in a power system, continuously track the load changes and endeavor to match the total power demand with total generation in such a manner that the operating cost is least. Conventional optimization techniques are cumbersome for such complex optimization tasks and are not suitable for on-line use due to increased computational burden. This paper proposes a neuro-fuzzy power dispatch method where the uncertainty involved with power demand is modeled as a fuzzy variable. Then Levenberg-Marquardt neural network (LMNN) is used to evaluate the optimal generation schedules. This model trains almost hundred times faster that the popular BP neural network. The proposed method (Hybrid Neuro Fuzzy System) has been tested on two test systems with six and thirteen generating units and found to be suitable for on-line economic dispatch.
\end{abstract}

Keywords: Economic dispatch (ED), Lambda iteration method, Gaussian membership functions, linguistic categories, Price penalty factor, Levenberg Marquardt algorithm, Hybrid Neuro Fuzzy System (HNFS)

\section{Introduction}

The basic objective of economic load dispatch of electric power generation is to schedule the committed generating unit outputs so as to meet the load demand at minimum operating cost while satisfying all units and system equality and inequality constraints. Main aspect of economic load dispatch is the on line economic dispatch where in it is required to distribute the load among the generating units actually paralleled with the system in such manner as to minimize the total cost of supplying the minute to minute requirement of the system. This problem is solved traditionally using mathematical programming based on optimization techniques such as lambda Iteration method, gradient method and dynamic programming method [1-3].

The classical lambda-iteration method has been used by power utilities for economic load dispatch (ELD). In Lambda iteration method convergence of the iterations is affected by the initial choice of lambda. Two types of iterations are involved in this method. First, lambda moves from its initial assumed value to its final optimal value iteratively. For systems with many generators, this movement can be oscillatory and may increase the computational time. Secondly, for each trial value of lambda, the associated generations have to be obtained using sub-iterations. Thus, the sub-iterations have to be involved many times. Therefore, this method could become too time consuming for effective real-time implementation.

Various artificial neural network based methods have been proposed for the ED problem [4-10]. Application of Hopfield method [4,5] and BP based methods [6-9] converge very slowly and suffer from local minima problem. Two phase neural network [9], Radial basis function [10] and Levenberg-Marquardt algorithms [11] have also been proposed as they do not suffer from these slow convergence problems. Recently there is an upsurge of hybrid methods [12-13] based on alternate approaches such as neural network and fuzzy logic due to their ability to model vague and noisy practical problems effectively. Approaches based on quadratic programming [14], fuzzy satisfaction maximizing technique [15], and genetic and evolutionary programming based hybrid approaches $[16,17]$ have been proposed for this problem.

In most of the common Multi-layer perceptron neural networks (MLP), the training is based on nonlinear optimization techniques. All these methods suffer from local minima problems, tend to converge very slowly and do not always achieve global minima. Several high performance algorithms are developed to train MLP models that converge 10 to 100 times faster than BP algorithm. These algorithms are based on numerical optimization techniques like conjugate gradient, quasi Newton and Levenberg-Marquardt algorithms. Out of these, Levenberg-Marquardt algorithm is found to be the fastest method for training moderate sized feedforward neural networks [18-19].

In this paper, a hybrid model has been developed for on-line economic dispatch which uses fuzzified inputs for training Levenberg-Marquardt neural network (LMNN).. In this paper, the efficiency of the hybrid LMNN (HNFS) has been demonstrated for the ED problem on 6 and 13-generating unit systems taken from reference [20]. 


\section{Methodology}

The block diagram of the proposed hybrid neuro-fuzzy approach is shown in Fig 1. A large number of loading patterns are generated in wide range of loads as shown in block I, and for each value of total power demand, the ED problem was solved using conventional lambda iteration method [1] to obtain optimal dispatch among the generating units [1], (block II). The generated power demand is fuzzified into different linguistic categories (block III). A large number of input-output patterns are thus generated to train the LMNN, taking fuzzified total power demand as the input. The trained LMNN (block IV) estimates the optimal power dispatches as well as operating cost for unknown patterns instantaneously.

\subsection{Optimal Generation Scheduling}

The objective of economic load dispatch is to minimize the total generation cost in a power system for a given load while satisfying various constraints. Thus economic dispatch is a constrained optimization problem and can be formulated as

Minimize the overall cost of generation

$$
\mathrm{C}=\sum_{i=1}^{m} C_{i}\left(P_{G i}\right)
$$

Subject to the inequality constraint

$$
P_{G i}^{\min } \leq P_{G i} \geq P_{G i}^{\max }, \quad \mathrm{i}=1,2,3 \ldots \ldots \ldots \mathrm{m} .
$$

and equality constraint of meeting the load demand with the transmission losses, i.e.

$$
\sum_{i=1}^{m} P_{G i}-\left(P_{D}-P_{L}\right)=0
$$

For a given real load $P_{D}$ at all the buses, the system $\operatorname{loss} P_{L}$ is a function of active power generation at each generating unit. To calculate system losses, two methods are in general use. One is the method of penalty factors and the other is the use of constant loss formula coefficients or B-coefficients [1,2]. The latter is commonly used by the power utilities and is adopted in this study. In this method, transmission losses are expressed as a quadratic function of generations:

$$
P_{L}=\sum_{i=1}^{m} \sum_{j=1}^{m} P_{i} B_{i j} P_{j}
$$

Fig.1 shows the block diagram of the economic optimal generation scheduling in a conventional power system. The main challenge for stable and reliable operation of an electric power system is to match the generation with the loads, under continuously varying system conditions including demand, supply and contingencies. The objective is to assign generations at connected generating units of the system in such a manner that the cost of operation is minimum while all the operating constraints are satisfied. This is a complex time intensive task for which optimization programs are run to compute the optimal values of real power generations every half an hour or so. When the optimal values are available, the appropriate control actions are taken through Automatic Generation Control (AGC) involving governor control and adjustment of valve setting adjusting fuel supply such that the units generate optimal allocated values.

\subsection{Hybrid Approach for ELD}

In the proposed hybrid approach, a large number of loading patterns were generated in wide range for the system under study. The generated patterns are fuzzified to include the uncertainty involved with power demand. Four fifth of the input-output pairs thus generated, are used to train the neural network. In order to speed up the neural network training, Levenberg-Marquardt BP algorithm is applied.

\subsection{Fuzzy modeling of load demand}

The power system load demand constantly changes and for each demand level optimum generation allocation is to be found. Development of probabilistic load models poses problems due to lack of proper and consistent field data. On the other hand fuzzy load data is presented in the form of possibility distributions of loads where the membership values can be derived from qualitative assessment, linguistic declarations, operator's past experiences or heuristics. In contrast to the conventional deterministic/ probabilistic load models, the proposed approach includes the stochastic behavior of loads by modeling them as fuzzy quantities having membership values in different linguistic categories. The incorporation of fuzziness in on-line applications is effective as the data available at the energy management center is normally vague, noisy and irrelevant. 


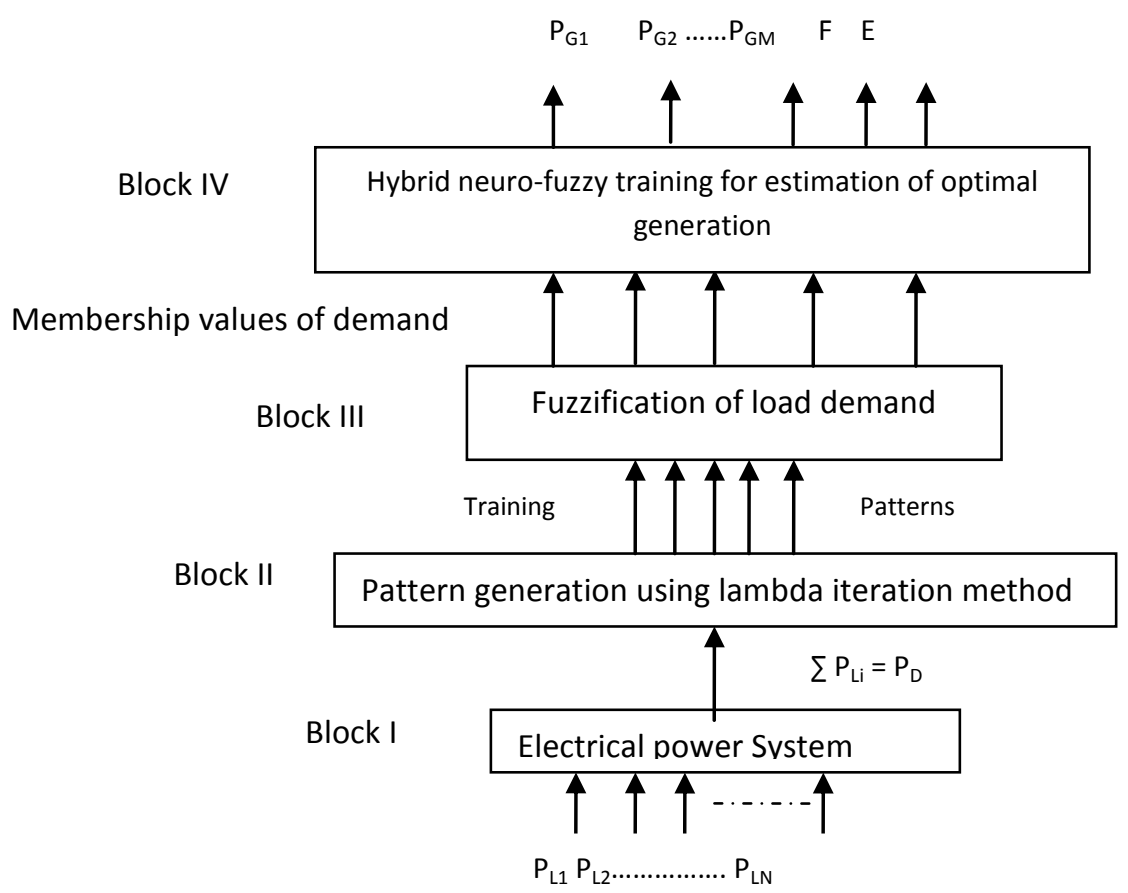

To model the uncertainty associated with load demand it is fuzzified into different linguistic categories. For the fuzzy representation of power demand, membership values in the range of 0 to 1 are assigned to each pattern generated. Thus crisp demand values are converted to fuzzy values. Load uncertainty is modeled by representing it as a fuzzy variable with memberships in different fuzzy linguistic categories, such as, very small (VS), small (S), medium (M), large (L) and very large (VL). The boundaries of these categories are fuzzified based on intuition and experience. Every load value in the crisp set is assigned membership values that represent the possibility of load being in that category. Non-linear guassian membership functions are used to find the membership values of $\mathrm{P}_{\mathrm{D}}$ in different fuzzy categories. In place of the more common triangular or trapezoidal functions, non-linear membership functions are found to be more suitable for representing power demand, as they ensure a smoother and more practical transition of loads from one category to the other. Also it does not add any complexity to the model. The membership value $(\mu)$ is calculated as [20]

$$
\mu_{i}=\frac{1}{1+\left[\frac{P_{D}-a_{i}}{b_{i}}\right]^{4}}
$$

$a_{i}$ and $b_{i}$ are parameters corresponding to linguistic category $i$ of power demand. These values can be chosen keeping in mind that $a_{i}$ represents the central value of the corresponding category, around which the membership value is equal to 1.0 and $b_{i}$, controls the width of the corresponding category. Heuristic and past experience play a key role in deciding the parameters $a_{i}$ and $b_{i}$,. Similarly the number of linguistic categories used for modeling may also vary from system to system. Thus by changing the parameters $a_{i}$ and $b_{i}$, different power systems can be modeled for any range of load variation. Table 2 and Table 7 show the fuzzification data used in this paper.

\subsection{Levenberg-Marquardt Neural Network}

In order to speed up the neural network training, Levenberg-Marquardt BP algorithm is applied. Various architectures of the LMNN models having different number of hidden nodes were trained for the same error goal and the optimal structure has been selected on the basis of the least training time. The trained LMNN has been found to be very fast and suitable for on-line generation dispatch as compared to the conventional methods which are slow and sometimes fail to converge.

The Levenberg-Marquardt algorithm is a variation of Newton's method [18]. This algorithm is very well suited to neural network training, where the performance index is the mean squared error and the variables $x$ are weights of the network. During supervised training a set of input patterns are presented to the network along with desired outputs (obtained using conventional method). The training is started with small random weights and the network is made to adjust the weights $x$ such that the difference between the network output and 
the target output is minimized. The mean squared error summed over $m$ number of output nodes, for PT number of patterns is defined as

$$
E R=\frac{1}{2 P T} \sum_{P T} \sum_{m}\left(T \arg e t_{m}-\text { Output }_{m}\right)^{2}
$$

Newton's update for optimizing mean squared error $E R(w)$ is

$$
w_{k+1}=w_{k}-A_{k}^{-1} g_{k}
$$

where $\left.A_{k} \equiv \nabla^{2} E R(w)\right|_{w=w_{k}}$ and $g_{k}=\left.\nabla E R(w)\right|_{w=w_{k}}$

As $E R(w)$ is a sum of square function it can be written as

$$
E R(w)=\sum_{P T}(T \arg e r-O u t p u t)^{T}(w)(T \arg e t-\text { Output })(w)
$$

then the $j^{\text {th }}$ element of the gradient would be

$$
[\nabla E R(w)]_{j}=\frac{\partial E R(w)}{\partial w_{j}}=2 \sum_{i=P T}(T \arg e t-\text { Output })_{i}(w) \frac{\partial(T \arg e t-\text { Output })_{i}(w)}{\partial w_{j}}
$$

The gradient can therefore be written in matrix form

$$
\nabla F(x)=2 J^{T}(w) v(x)
$$

where $\mathbf{J}$ is the Jacobean matrix giving sensitivity. Next, the Hessian matrix is to be determined. The $\mathrm{k}, \mathrm{j}$ element

of the Hessian matrix would be $\left[\nabla^{2} \boldsymbol{E R}(w)\right]_{k j}=\frac{\partial^{2} E R(w)}{\partial w_{k} \partial w_{j}}$

The Hessian matrix can then be expressed in matrix form

$\nabla^{2} E R(w)=2 J^{T}(w) J(w)+2 S(w)$

where $S(w)=\sum_{i=1}^{N}(T \arg \text { et }- \text { Output })_{i}(w) \nabla^{2}(T \arg \text { et }- \text { Output })_{i}(w)$

If $S(w)$ is assumed to be small, the Hessian matrix can be approximated as

$\nabla^{2} F(w) \cong 2 J^{T}(w) J(w)$

Substituting (10) and (14) into (7), the Gauss-Newton method is obtained as

$$
w_{k+1}=w_{k}-\left[2 J^{T}\left(w_{k}\right) J\left(w_{k}\right)\right]^{-1} 2 J^{T}\left(w_{k}\right) v\left(w_{k}\right)
$$

From this, it is evident that the advantage of Gauss-Newton method over the standard Newton's method is that it does not require calculation of second -order derivatives. One problem with the Gauss-Newton method is that the matrix $\mathrm{H}=\mathbf{J}^{\mathrm{T}} \mathrm{J}$ may not be invertible. This can be overcome by using the following modification to the approximate Hessian matrix.

$$
G=H+\mu I
$$

To make this matrix invertible, suppose that the eigen values and eigen vectors of $\mathrm{H}$ are $\left\{\lambda_{1}, \lambda_{2} \ldots . ., \lambda_{n}\right\}$ and $\left\{z_{1}, z_{2}, \ldots \ldots, z_{n}\right\}$

Then $\boldsymbol{G} z_{i}=[\boldsymbol{H}+\boldsymbol{\mu I}] z_{i}=\boldsymbol{H} z_{i}+\boldsymbol{\mu} z_{i}=\lambda_{i} z_{i}+\mu z_{i}$

Therefore the eigenvectors of $\mathrm{G}$ are the same as the eigenvectors of $\mathrm{H}$ and the eigen values of $\mathrm{G}$ are $\left(\left(\lambda_{i}+\mu\right)\right.$.

G can be made positive definite by increasing $\mu$ until $\left(\lambda_{i}+\mu\right)>0$ for all $\mathrm{i}$, and therefore the matrix will be invertible. This leads to the Levenberg-Marquardt algorithm [18].

$$
\Delta w_{k}=-\left[J^{T}\left(w_{k}\right) J\left(w_{k}\right)+\mu_{k} I\right]^{-1} J^{T}\left(w_{k}\right) v\left(w_{k}\right)
$$

This algorithm has a useful feature that as $\mu_{k}$ is increased it approaches the steepest descent algorithm with small learning rate

$$
w_{k+1} \cong w_{k}-\frac{1}{\mu_{k}} J^{T}\left(w_{k}\right) v\left(w_{k}\right)
$$

and if $\mu_{k}$ is decreased to zero the algorithm becomes Gauss-Newton. The algorithm begins with $\mu_{k}$ set to some small value. 


\section{Results and Discussion}

The effectiveness of the proposed hybrid approach for economic dispatch problem has been demonstrated on two test systems. It has been found that the LMNN trains very fast (in a few iterations) due to the effective Levenberg-Marquardt algorithm. In comparison to slow converging conventional methods this method is highly suitable for determining on-line dispatch strategy accurately in modern market driven power systems. Using this algorithm, it is easy to decide the optimum neural network architecture for a given error goal.

\subsection{Six Generating Unit System}

The cost coefficient and generation limits of six-unit system are given in Table 1. Transmission losses are calculated using B matrix [1] in Table 3. To establish the effectiveness of developed LMNN for ED, the neural network models are trained for 6-generating unit system. The load patterns are generated by varying the load at each bus of the system randomly. The conventional lambda iteration method was applied for each load pattern to obtain the optimum value of real power at different generating units, for minimum cost .Total 300 patterns were generated by changing load $\left(\mathrm{P}_{\mathrm{D}}\right)$ between $900 \mathrm{MW}$ to $1350 \mathrm{MW}$. The generated patterns were fuzzified using eq. (6) and data in Table 3. The values in Table 4 are selected based on operator experience or expert judgment. The fuzzification of loads is represented in Fig. 2. Then 240 fuzzified patterns were used for trainings LMNN while remaining 60 patterns were used for testing the performance of the trained network. The LMNN is also capable of producing the minimum cost content corresponding to the optimal solution.

Table 1.Cost Coefficients and Generation limits of Six-unit system

\begin{tabular}{cccccc}
\hline S. No. & $P_{i \min }$ & $P_{i \max }$ & $a_{i}$ & $\mathrm{~b}_{\mathrm{i}}$ & $\mathrm{c}_{\mathrm{i}}$ \\
\hline 1 & 100 & 500 & 0.007 & 7 & 200 \\
2 & 50 & 200 & 0.0095 & 10 & 200 \\
3 & 80 & 300 & 0.009 & 8.5 & 220 \\
4 & 50 & 150 & 0.009 & 11 & 200 \\
5 & 50 & 200 & 0.008 & 10.5 & 220 \\
6 & 50 & 120 & 0.0075 & 12 & 190 \\
\hline
\end{tabular}

Table 2.Data for Fuzzy Modeling of Power Demand for 6-unit System

\begin{tabular}{cccccc}
\hline $\begin{array}{c}\text { Linguistic } \\
\text { Category for } \mathrm{P}_{\mathrm{D}}\end{array}$ & $\begin{array}{c}\text { Very } \\
\text { Small }\end{array}$ & Small & Medium & Large & $\begin{array}{c}\text { Very } \\
\text { Large }\end{array}$ \\
\hline$a_{i}$ & 900 & 1000 & 1100 & 1200 & 1300 \\
$\mathrm{~b}_{\mathrm{i}}$ & 50 & 60 & 70 & 60 & 65 \\
\hline
\end{tabular}

Table 3.B-coefficients of Six-unit system

\begin{tabular}{rrrrrr}
\hline 0.0017 & 0.0012 & 0.007 & -0.0001 & -0.0005 & -0.0002 \\
0.0012 & 0.0014 & 0.0009 & 0.0001 & -0.0006 & -0.0001 \\
0.0007 & 0.0009 & 0.0031 & 0 & -0.001 & -0.0006 \\
-0.0001 & 0.0001 & 0 & 0.0024 & -0.0006 & -0.0008 \\
-0.0005 & -0.0006 & -0.001 & -0.0006 & 0.0129 & -0.0002 \\
-0.0002 & -0.0001 & -0.0006 & -0.0008 & -0.0002 & 0.015 \\
\hline
\end{tabular}

The developed LMNN has five input nodes (membership values of $P_{D}$ in five fuzzy categories of very low, medium etc) and 7 output nodes ( 6 nodes for unit outputs, one for incremental fuel cost). The optimum size of the neural network has been obtained by training LMNN models having different no. of hidden nodes for the training error goal of $1 \times 10^{-3} \mathrm{pu}$. The training performance of the neural networks having different structures has been shown in Table 4. As can be observed from Table 4, the LMNN model having 18 hidden nodes (5-18-7) is the most efficient structure, as it required the least training time for the same error goal.

Table 4.Training Performance of Hybrid LMNN (HNFS) with different structures for 6-units system 


\begin{tabular}{cccc}
\hline S.No & No. of Hidden nodes & Training time & No. of Epochs \\
\hline 1 & 11 & $1.0 \mathrm{~s}$ & 6 \\
$\mathbf{2}$ & $\mathbf{1 8}$ & $\mathbf{. 2 5} \mathrm{s}$ & $\mathbf{5}$ \\
3 & 20 & $.7 \mathrm{~s}$ & 15 \\
4 & 24 & $.4 \mathrm{~s}$ & 7 \\
5 & 28 & $2.1 \mathrm{~s}$ & 33 \\
\hline
\end{tabular}

The testing performance (percentage testing error) of the trained LMNN for all the 60 testing patterns is compared with conventional method and plotted in Fig. 3-Fig. 9. The figures clearly show that the trained neural network produces accurate results i.e. almost negligible error for all the testing patterns for all the seven outputs (generations, incremental fuel cost (lambda) ). Result of about 8 testing patters have been compared in Table 5 and Table 6 with conventional results to show that the developed hybrid approach(HNFS) is capable of computing the real power generations and incremental cost.

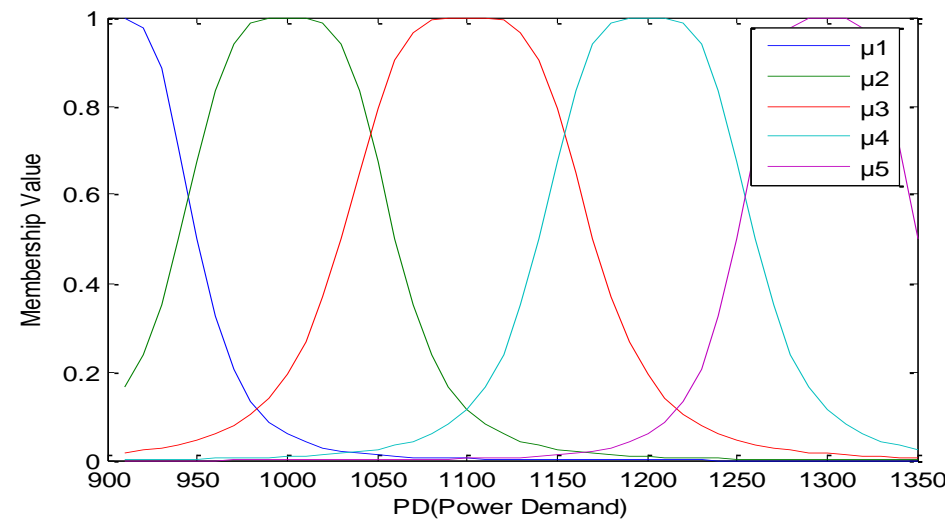

Fig. 2. Fuzzy modeling of power demand for ED (6-unit system)

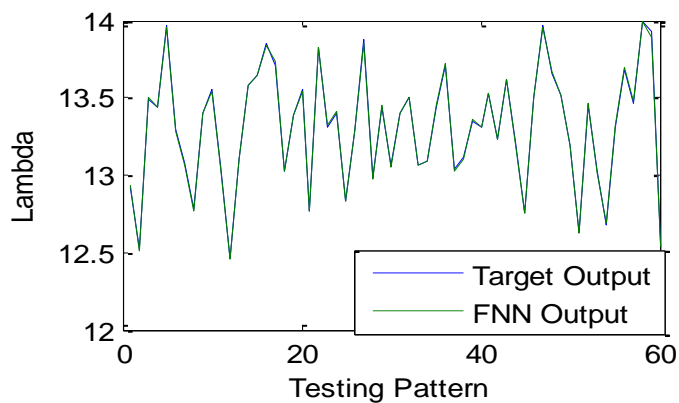

Fig. 3.Results for Lambda of 6-Generating unit System

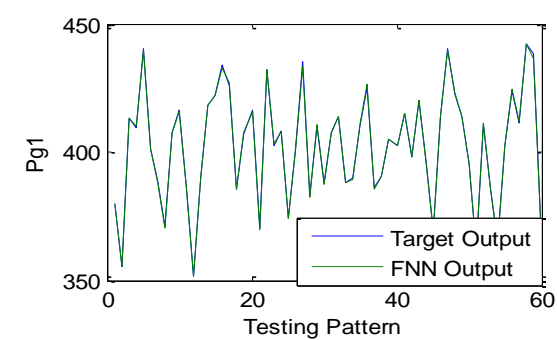

Fig. 4.Results for power allocation of unit-one of 6-Generating unit System

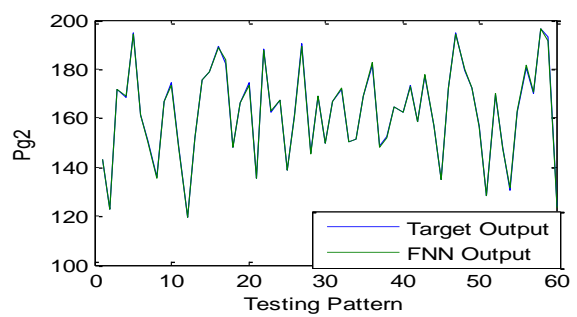


Fig. 5.Results for power allocation of unit-two of 6-Generating unit System

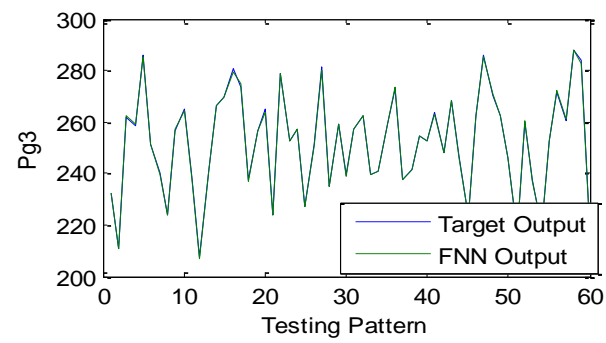

Fig. 6.Results for power allocation of unit-three of 6-Generating unit System

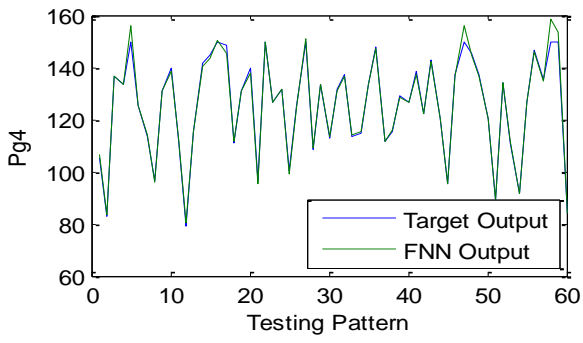

Fig. 7.Results for power allocation of unit-four of 6-Generating unit System

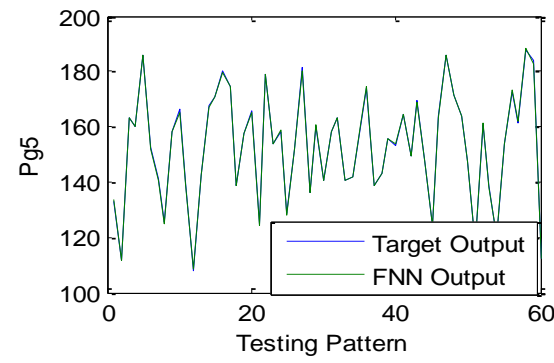

Fig. 8.Results for power allocation of unit-five of 6-Generating unit System

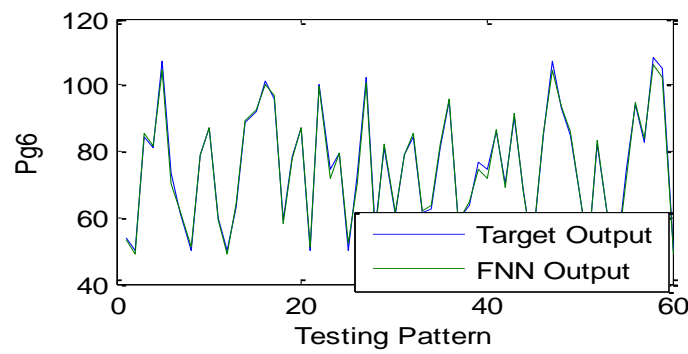

Fig. 9.Results for power allocation of unit-six of 6-Generating unit System

Table 5.Testing Performance of The (5-18-7) hybrid LMNN (HNFS)

\begin{tabular}{|c|c|c|c|c|c|c|c|c|c|}
\hline \multirow[t]{2}{*}{ S.no. } & \multirow{2}{*}{$\begin{array}{l}\text { Output } \\
\text { value }\end{array}$} & \multicolumn{2}{|c|}{$\mathrm{PD}=902.513$} & \multicolumn{2}{|c|}{$\mathrm{PD}=951.576$} & \multicolumn{2}{|c|}{$\mathrm{PD}=1014.646$} & \multicolumn{2}{|c|}{$\mathrm{PD}=1067.224$} \\
\hline & & Classical & HNFS & Classical & HNFS & Classical & HNFS & Classical & HNFS \\
\hline 1. & Lambda & 12.445 & 12.446 & 12.631 & 12.633 & 12.866 & 12.865 & 13.033 & 13.035 \\
\hline 2. & Pg1 & 351.091 & 351.099 & 362.218 & 362.228 & 376.242 & 376.250 & 386.103 & 386.113 \\
\hline 3. & $\mathrm{Pg} 2$ & 119.257 & 119.265 & 128.554 & 128.563 & 140.264 & 140.272 & 148.549 & 148.558 \\
\hline 4. & $\operatorname{Pg} 3$ & 206.879 & 206.888 & 216.572 & 216.582 & 229.126 & 229.137 & 237.825 & 237.832 \\
\hline 5. & $\operatorname{Pg} 4$ & 78.848 & 78.857 & 88.959 & 88.942 & 102.192 & 102.198 & 111.306 & 111.317 \\
\hline 6. & $\operatorname{Pg} 5$ & 107.715 & 107.722 & 117.603 & 117.609 & 130.098 & 130.107 & 138.871 & 138.879 \\
\hline 7. & Pg6 & 50.000 & 50.010 & 50.000 & 50.005 & 50.483 & 50.486 & 59.465 & 59.469 \\
\hline
\end{tabular}

Table 6.Testing Performance of The (5-18-7) hybrid LMNN (HNFS) 
Hybrid Neuro-Fuzzy System for Optimal Generation scheduling in Electrical Power systems

\begin{tabular}{llllllllll}
\hline S.no. & $\begin{array}{l}\text { Output } \\
\text { value }\end{array}$ & \multicolumn{2}{l}{$\mathrm{PD}=1100.293$} & \multicolumn{2}{c}{$\mathrm{PD}=1162.256$} & \multicolumn{2}{c}{$\mathrm{PD}=1249.381$} & \multicolumn{2}{c}{$\mathrm{PD}=1332.736$} \\
\cline { 3 - 9 } & & Classical & HNFS & Classical & HNFS & Classical & HNFS & Classical & HNFS \\
\hline 1. & Lambda & 13.138 & 13.139 & 13.336 & 13.339 & 13.615 & 13.617 & 13.928 & 13.930 \\
2. & Pg1 & 392.305 & 392.314 & 403.926 & 403.934 & 420.264 & 420.272 & 438.075 & 438.082 \\
3. & Pg2 & 153.771 & 153.780 & 163.579 & 163.585 & 177.421 & 177.429 & 193.084 & 193.093 \\
4. & Pg3 & 243.308 & 243.317 & 253.604 & 253.612 & 268.133 & 268.141 & 284.301 & 284.309 \\
5. & Pg4 & 117.053 & 117.062 & 127.852 & 127.861 & 143.106 & 143.113 & 150.000 & 150.008 \\
6. & Pg5 & 144.389 & 144.396 & 154.730 & 154.739 & 169.273 & 169.279 & 184.054 & 184.059 \\
7. & Pg6 & 65.109 & 65.118 & 75.676 & 75.684 & 90.511 & 90.518 & 105.091 & 105.099 \\
\hline
\end{tabular}

\subsection{Thirteen Generating Unit System}

The cost coefficient data along with generating limits for the Thirteen-unit power system i and Bcoefficients have been taken [10]. In this case also, 300 load patterns were generated, changing the load at different buses randomly between 2000MW and $2400 \mathrm{MW}$ in wide range. The generated loads were fuzzified using eq (6) and the data selected for fuzzification which is given in Table 7. The fuzzified demand for the full range is plotted in Figure 10 showing the memberships in all five categories. Out of 300 patterns, 240 patterns were used for training using Levenberg-Marquardt algorithm based feed forward network, while the remaining 60 patterns were used for the testing purpose.

Table 7.Data for fuzzy modeling of power demand for 13-unit system

\begin{tabular}{cccccc}
\hline $\begin{array}{c}\text { Linguistic Category } \\
\text { for } \mathrm{P}_{\mathrm{D}}\end{array}$ & Very Small & Small & Medium & Large & Very Large \\
\hline$a_{i}$ & 2000 & 2090 & 2180 & 2270 & 2360 \\
$\mathrm{~b}_{\mathrm{i}}$ & 60 & 60 & 70 & 60 & 65 \\
\hline
\end{tabular}

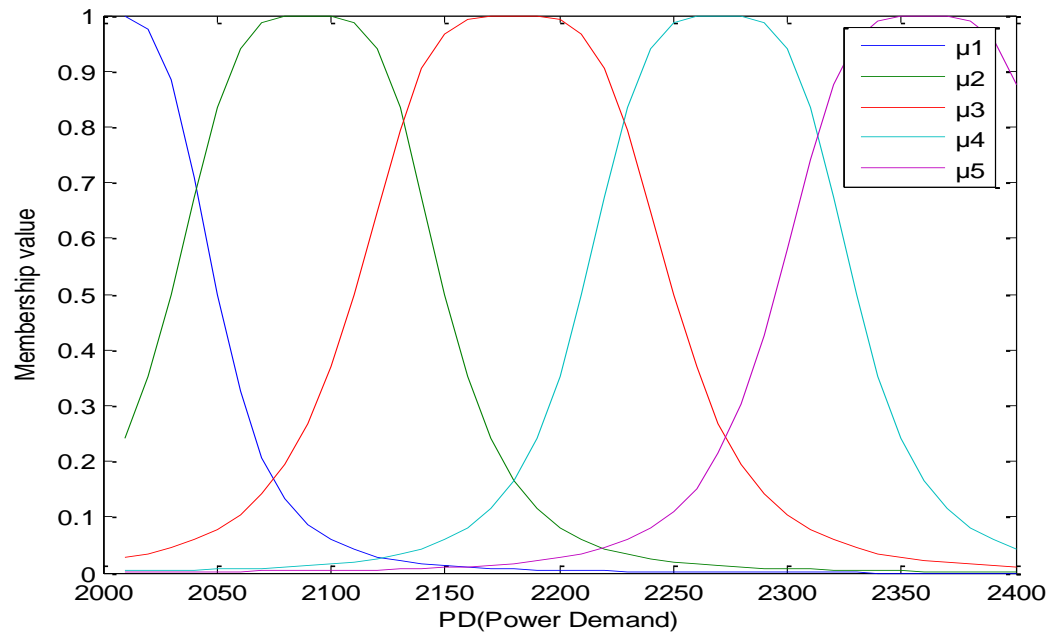

Fig. 10. Fuzzy modeling of power demand for ED (13-unit system)

Table 8.Training Performance of hybrid LMNN for different structures for 13-unit system

\begin{tabular}{cccc}
\hline S.No. & No. of Hidden nodes & Training time & No. of Epochs \\
\hline 1 & $\mathbf{1 1}$ & $\mathbf{0 . 6 ~ s}$ & $\mathbf{8}$ \\
2 & 19 & $1.2 \mathrm{~s}$ & 14 \\
3 & 22 & $1.5 \mathrm{~s}$ & 12 \\
4 & 25 & $1.7 \mathrm{~s}$ & 12 \\
5 & 30 & $3.6 \mathrm{~s}$ & 20 \\
\hline
\end{tabular}


After training different structures for different number of hidden nodes for the training error goal of $1 \times 10^{-3} \mathrm{pu}$, the optimum size of LMNN for 13-unit economic dispatch problem was found to be (5-11-14). The training performance of the neural networks having different structures has been shown in Table 8. The results of the trained neural network for the previously unseen 60 patterns have been shown in Fig 11-Fig 24. The testing results of 4 patterns are shown in Table 9 and are compared with those obtained from classical lambda iteration method. It can be seen that the proposed LMNN based hybrid method (Hybrid Neuro Fuzzy System) is accurate and trains very fast without facing local minima problems.

Table 9.Testing Performance off The (5-11-14) hybrid LMNN (HNFS)

\begin{tabular}{rlcccccccc}
\hline S.no. & Out Put & \multicolumn{2}{c}{$\mathrm{PD}=2004.772$} & \multicolumn{2}{c}{$\mathrm{PD}=2240.795$} & \multicolumn{2}{c}{$\mathrm{PD}=2349.380$} & \multicolumn{2}{c}{$\mathrm{PD}=2384.915$} \\
\cline { 2 - 9 } & Value & Classical & HNFS & Classical & HNFS & Classical & HNFS & Classical & HNFS \\
\hline 1 & Lambda & 9.153 & 9.155 & 9.498 & 9.496 & 9.488 & 9.489 & 9.546 & 9.547 \\
2 & Pg1 & 60 & 60 & 60 & 60 & 60 & 60 & 60 & 60 \\
3 & Pg2 & 60 & 60.003 & 60 & 60.006 & 94.566 & 94.569 & 101.806 & 101.8 \\
4 & Pg3 & 60 & 60.002 & 106.16 & 106.17 & 101.02 & 101.03 & 108.795 & 108.8 \\
5 & Pg4 & 60 & 60.002 & 99.564 & 99.569 & 93.637 & 93.638 & 100.802 & 100.8 \\
6 & Pg5 & 60 & 60.004 & 60 & 60.002 & 90.769 & 90.762 & 97.696 & 97.7 \\
7 & Pg6 & 60 & 60.002 & 102.07 & 102.07 & 96 & 96.006 & 103.366 & 103.4 \\
8 & Pg7 & 82.713 & 82.715 & 120 & 120.01 & 120 & 120.01 & 120 & 120 \\
9 & Pg8 & 80.246 & 80.242 & 120 & 120 & 120 & 120 & 120 & 120 \\
10 & Pg9 & 83.927 & 83.93 & 120 & 120 & 120 & 120 & 120 & 120 \\
11 & Pg10 & 55 & 55.003 & 55 & 55.004 & 120 & 120.01 & 120 & 120 \\
12 & Pg11 & 680 & 680 & 680 & 680 & 680 & 680 & 680 & 680 \\
13 & Pg12 & 360 & 360 & 360 & 360 & 360 & 360 & 360 & 360 \\
14 & Pg13 & 360 & 360 & 360 & 360 & 360 & 360 & 360 & 360 \\
\hline
\end{tabular}

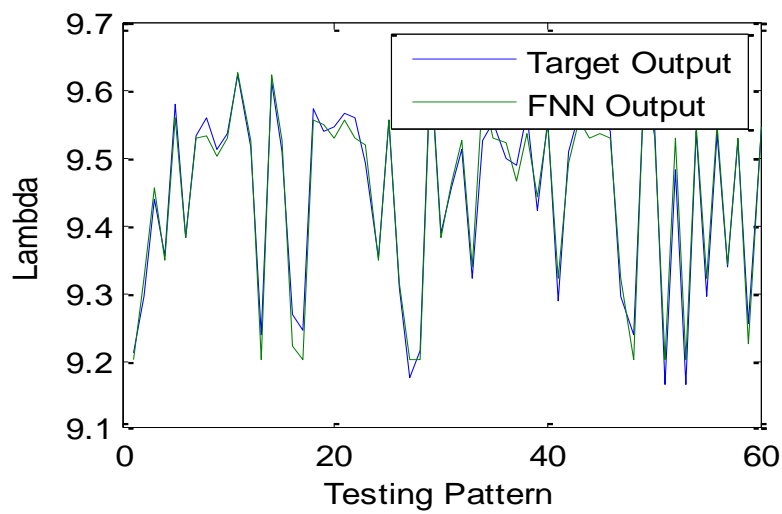

Fig. 11.Results for Lambda of 13-Generating unit System

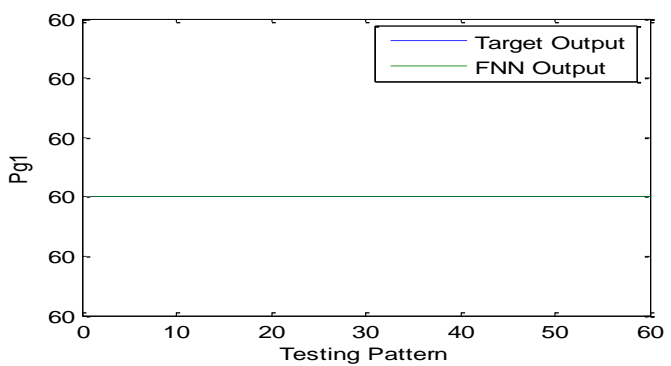

Fig. 12.Results for cost of operation for unit one of 13-Generating unit System 


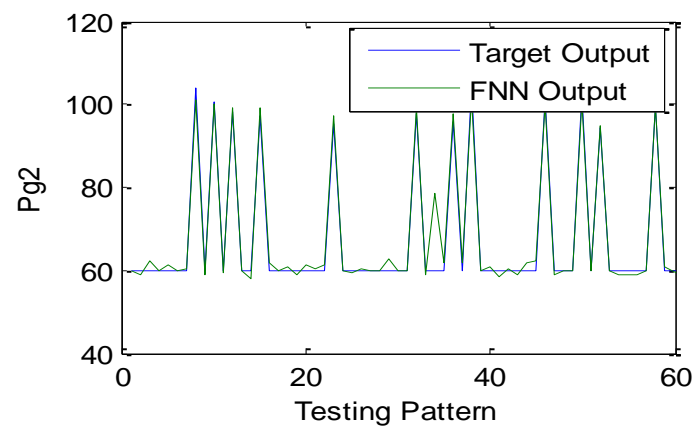

Fig. 13.Results for cost of operation for unit two of 13-Generating unit System

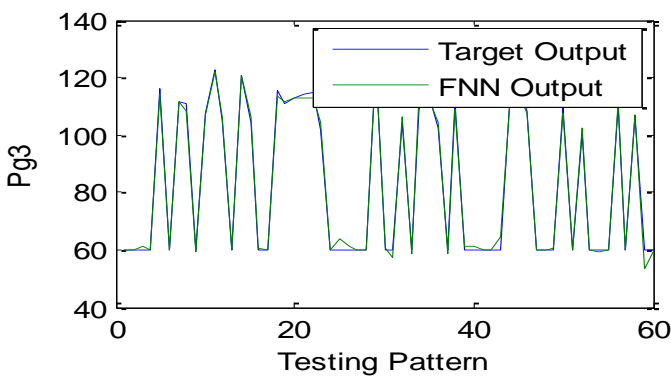

Fig. 14.Results for cost of operation for unit three of 13-Generating unit System

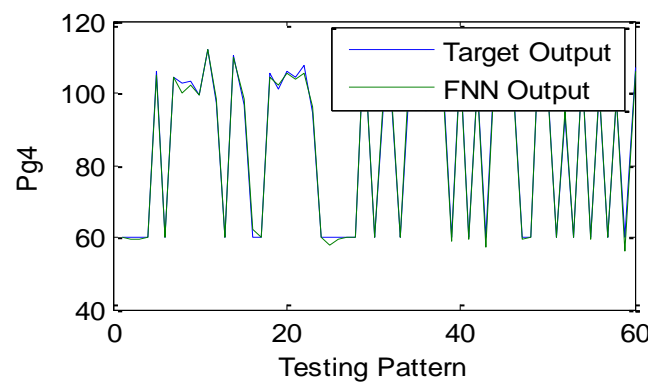

Fig. 15.Results for power generation for unit four of 13-Generating unit System

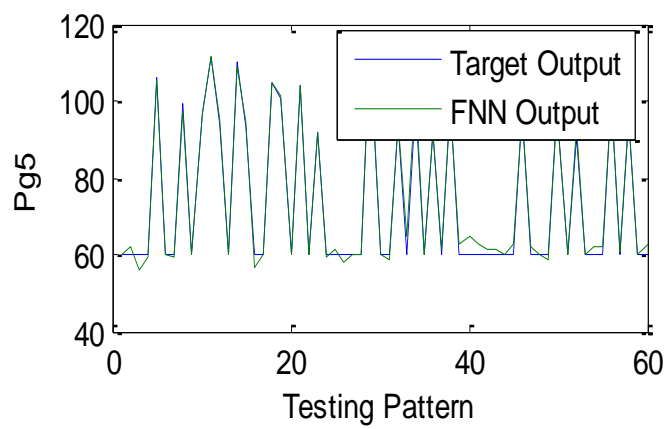

Fig. 16.Results for cost of operation for unit five of 13-generating unit system

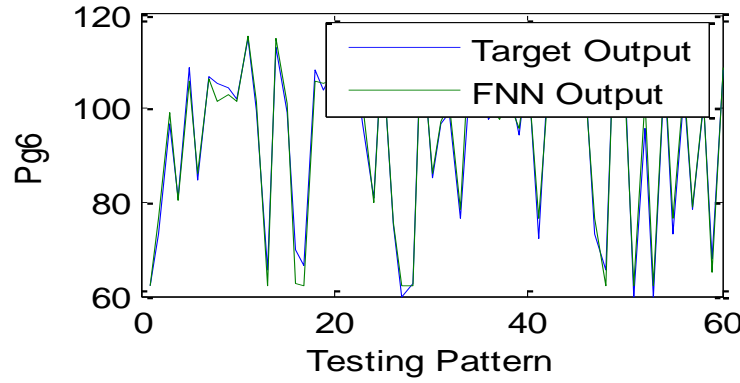

Fig. 17. Results for cost of operation for unit six of 13 -generating unit system 


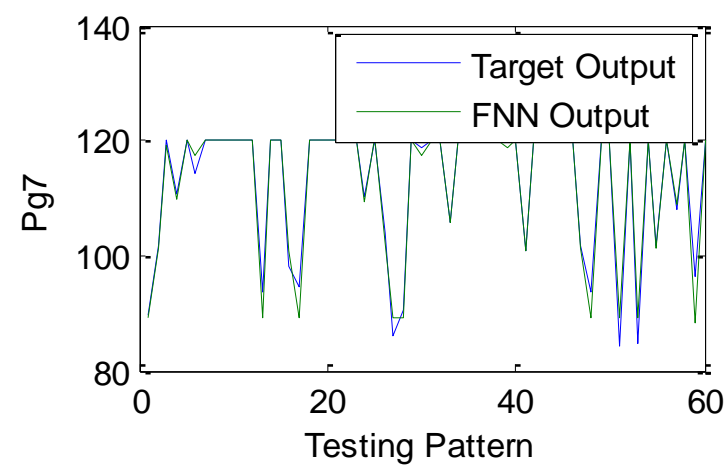

Fig.18. Results for cost of operation for unit seven of 13-generating unit system

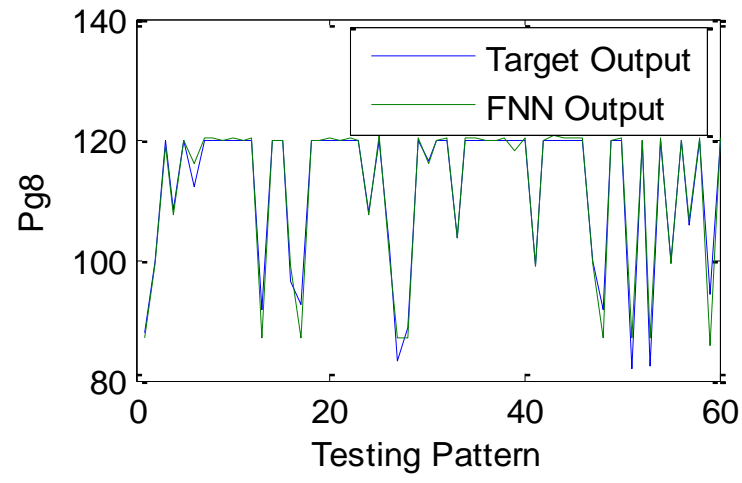

Fig.19. Results for cost of operation for unit eight of 13-generating unit system.

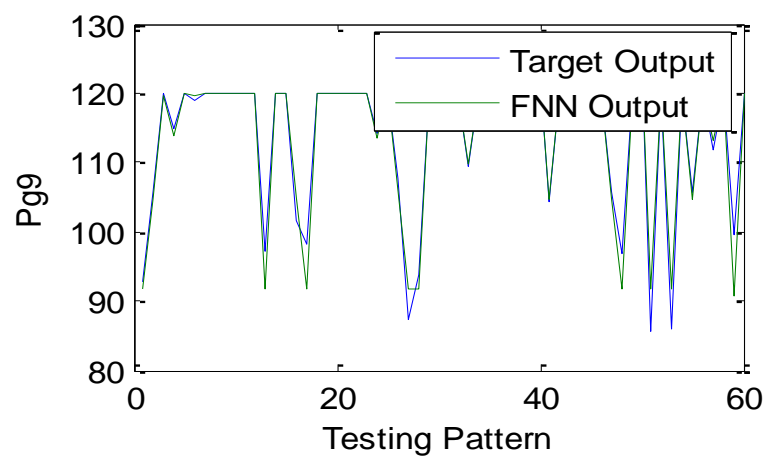

Fig.20. Results for cost of operation for unit nine of 13-generating unit system.

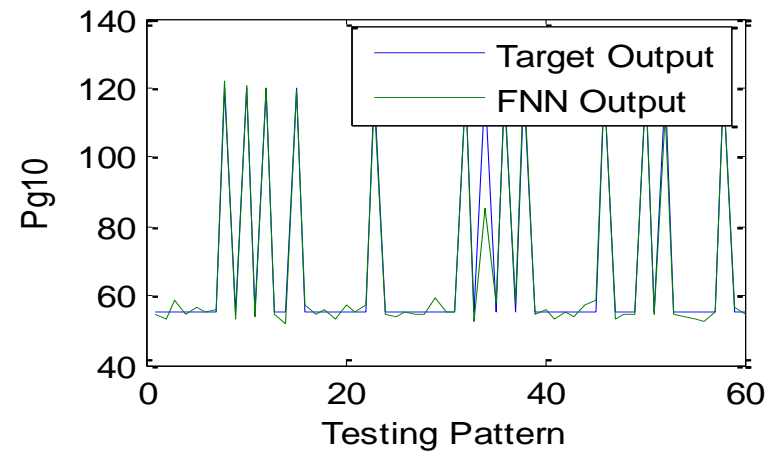

Fig.21. Results for cost of operation for unit ten of 13 -generating unit system 


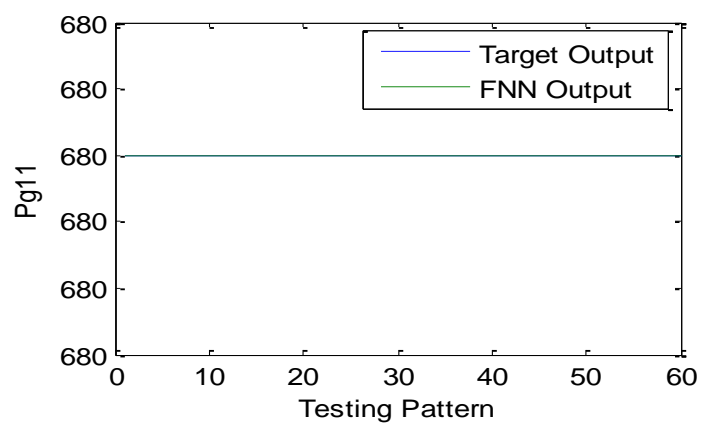

Fig.22. Results for cost of operation for unit eleven of 13-generating unit system.

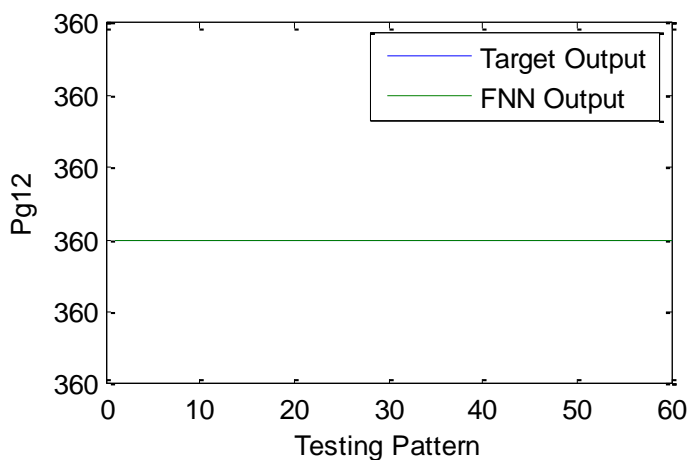

Fig.23. Results for cost of operation for unit twelve of 13-generating unit system.

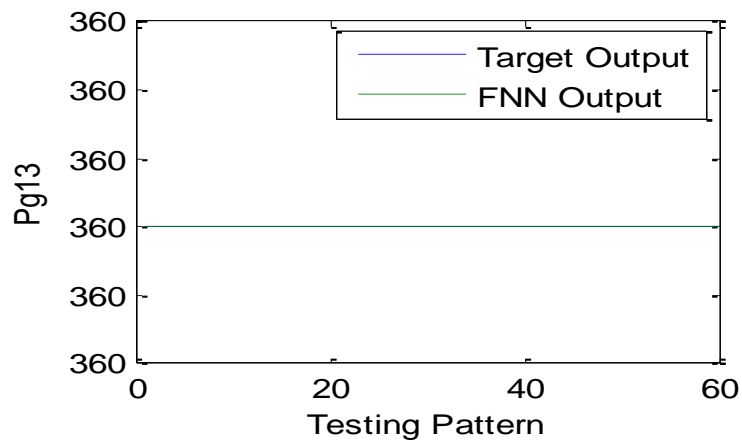

Fig.24. Results for cost of operation for unit thirteen of 13-generating unit system.

\section{Conclusion}

The paper proposes an efficient hybrid method for handling the complex problem of optimum generation allocation for constantly changing loads, keeping operating cost at their least level. Classical lambda iteration method for economic load dispatch problem for a practical power system is found to be too time consuming for effective real-time implementation.

The proposed hybrid Levenberg-Marquardt based neural network (HNFS) is trained to provide the optimal value of incremental cost and economic generation dispatch on all the committed generating units for a given power demand. Due to the fuzzification of input power demand the network is expected to perform well under practical conditions where the data may be vague, noisy or incorrect. During testing phase, the trained hybrid network provided accurate results for previously unseen patterns. The proposed method is noise tolerant and can adapt to changing system conditions very easily. As the training of the LMNN using LevenbergMarquardt algorithm is extremely fast, it may be used for on-line implementation at energy management centre.

\section{Acknowledgements}

The authors sincerely acknowledge the financial support provided by University Grant Commission (UGC), New Delhi, India under research project entitled on "Application of soft Computing techniques in power system optimization" vide letter no. F.No. 41-661/2012(SR) dated 23/7/2012. The authors also thank the Honorable V.C., R.G.T.U. Bhopal for providing facilities for carrying out this work. 


\section{References}

[1] L.K. Kirchmayer, Economic Opertion of power system (New York: Wiley,1958)

[2] A.J. Wood and B.F. Wollenberg, Power Generation, Operation and Control ( New York: Wiley, 1984.)

[3] Hadi Sadat, Power System Analysis( Tata McGraw Hill, International Edition, 1999).

[4] J.H. Park, Y.S. Kim, I.K. Eom, and K.Y. Lee, "Economic load dispatch for piecewise quadratic cost function using Hopfield neural network " IEEE Trans Power Syst., vol. 8(3), pp.1030-1035, 1993.

[5] C.T. Su and G.J.Chiou," A fast computation Hopfield method to economic load dispatch of power systems", IEEE Trans Power Syst., vol,12. No. 4, pp. 1759-1764, 1997.

[6] P.S. Kulkarni, A.G. Kothari and D.P.Kothari, "Combined economic and emission dispatch using improved back propagation neural network", International Journal of Electric Machines and Power Systems, vol. 28,pp. 31-43, 2000.

[7] G. Singh, S.C. Srivastava, P.K. Kalra and D.M. Vinod Kumar, "Fast approach to artificial neural network training and its application to economic load dispatch", Electric machines and power systems, pp. 13-24, 1995.

[8] P.K. Hota, R. Chakrabarty and P.K.Chattopadhyay, "An integrated approach to economic emission load dispatch using neural network and goal attainment methods", Electric machines and power systems, pp. 1085-1095, 1999.

[9] R. Naresh, J. Dubey and J.D. Sharma, "Two Phase neural network based frame work modelling of constraind economic load dispatch", IEE proceedings Gener Trans. Dist, vol. 151, no. 3, 2004, pp373 -380.

[10] P. Arvindbabu and K.R. Nayar, "Economic dispatch based on optimal lambda using radial basis function network", Electrical Power and Energy Systems, vol. 24, pp. 551-556, 2002.

[11] Krishna Teerth Chaturvedi, Laxmi Srivastava, and Manjaree Pandit, "Levenberg Merquardt algorithm based optimal load dispatch", IEEE Power India Conference 2006, New Delhi 10-12 $2^{\text {th }}$ April 2006.

[12] Manjaree Pandit, Laxmi Srivastava and J. Sharma, "Fast voltage contingency selection using fuzzy Parallel Self-organizing Hierarchical Neural Network", IEEE Trans Power Syst, vol.18, No. 2, pp. 657-664. May 2003.

[13] Krishna Teerth Chaturvedi, Manjaree Pandit and Laxmi Srivastava, Jaydev Sharma and R.P. Bhatele, "Hybrid Fuzzy-Neural Network Based Composite Contingency Ranking Employing Fuzzy Curves for Feature Selection", Neurocomputing (Elsevier) vol. 73, pp. 506-516, 2009 .

[14] R.M.S. Danaraj and Dr. F. Gajendran, “Quadratic programming solution to emission and economic dispatch problems”, Journal of Inst. of Engineers, (India), pt. El., vol. 86, pp. 129-132, September 2005

[15] Chao- Ming Haung, Hong-tzer Yang, and Ching-Lien Huang, " Bi- Objective power Dispatch Using fuzzy satisfactionmaximizing decision approach", IEEE Trans. Power syst, vol. 12, no. 4, pp.1715-1721, Nov 1997.

[16] Venkatesh P, Gnanadass R and Padhy Narayana Prasad, "Comparision and application of evolutionary programming techniques to combined economic emission dispatch with line flow constraint”. IEEE Trans Power Syst. 18(2):688-97, 2003.

[17] D.N. Jeyakumar, T.Jayabarathi, and T. Raghunathan, "Particle swarm optimization for various types of economic dispatch problems", Electrical Power and Energy Syst, vol. 28, pp. 36-42, 2006.

[18] M.T. Hagan, and M.H. Mehnaj, "Training feed-forward neural networks with Marquard algorithm," IEEE Trans. On Neural Network,vol. 5(6), pp. 989-993, 1994.

[19] L. M. Saini, and M.K. Soni, “Artificial neural network based peak load forecasting using Levenberg-Marquardt and quasi-Newton method, " IEE Proceeding Gener. Trans. Distrib., pp.578-584, 2002

[20] K.H. Abdul-Rahman, S.M. Shahidehpour, and M. Daneshdoost, "AI approach to optimal VAR control with fuzzy reactive loads", IEEE Trans. Power Systs, vol. 10, pp. 88-97, Feb.1995. 